\title{
Relationship between Early Exposure to Tobacco Smoke and Intima Media Thickness (IMT) in COPD patients
}

\author{
Akinori EbIHARA $^{1}$ Asuka NAGAI $^{1}$ Rurika HAMANAKA ${ }^{2}$ Naoko IMAMURA $^{2}$ \\ Chizumi YAMADA $^{3}$ Tokuzen IWAMOTO $^{1}$ Ichiro KUWAHIRA $^{1}$
}

${ }^{1}$ Pulmonary Medicine, Tokai University Tokyo Hospital

${ }^{2}$ General Thoracic Surgery, Tokai University Tokyo Hospital

${ }^{3}$ Internal Medicine, Tokai University Tokyo Hospital

\begin{abstract}
Background We have previously reported that early exposure to tobacco smoke significantly increases the prevalence of chronic obstructive pulmonary disease (COPD). As a follow-up, we hypothesized that COPD arising from early exposure to tobacco smoke may increase intima media thickness (IMT), which correlates with an increased prevalence of smoke-related vascular comorbidities such as cardiovascular and cerebrovascular diseases.

Methods This prospective, observational study of a consecutive cohort of COPD patients was carried out between 2009 and 2013. Potential subjects were identified from the Erimo town clinic and were divided into three groups: Group (1) had a history of COPD and early exposure to tobacco smoke; Group (2) had a history of COPD and non-early exposure to tobacco smoke; and Group (3) included subjects without COPD. The IMT of members of all 3 groups was measured by ultrasonography using the longitudinal axis of the common carotid arteries. Early smoke exposure was defined as habitual smoking starting before age 20 .

Results A total of 152 subjects (mean age $72 \pm 10$ years) were enrolled in the study after providing their informed consent. Groups 1, 2, and 3 consisted of 41 subjects (mean age $68 \pm 9$ ), 80 subjects (mean age $71 \pm 11$ ), and 31 subjects (mean age $69 \pm 10$ ), respectively. The maximum IMT value in Groups 1, 2, and 3 was $1.62 \pm 0.35 \mathrm{~mm}, 1.46 \pm 0.43 \mathrm{~mm}$, and $1.32 \pm 0.26 \mathrm{~mm}$, respectively. Groups with a history of COPD tended to have higher maximum IMT values that the group without COPD. Furthermore, Group 1 had the highest IMT value.

Conclusion In subjects with COPD, early smoke exposure promoted atherosclerotic changes, which may have increased the likelihood of smoke-related vascular comorbidities such as cardiovascular and cerebrovascular diseases. Further studies are needed to elucidate the precise magnitude of the increased risk of these comorbidities associated with COPD and early smoke exposure. (HEP. 2014; 41: 524-527.)
\end{abstract}

Key words Early exposure, tabacco smoke, COPD, Intima Media Thickness, vascular comorbidities

\section{Introduction}

In a survey by the Ministry of Health, Labour and Welfare of Japan in 2012, chronic obstructive pulmonary disease (COPD) was identified as the ninth leading cause of death in Japan. In addition, an epidemiological study by the World Health Organization (WHO) has predicted that COPD will become the third leading cause of death in $2020^{1)}$. Comorbidities and systemic complications are particularly problematic for COPD patients, and COPD is positioned as a systemic disease with various complications ${ }^{2)}$. Many comorbidities associated with aging and smoking are seen in COPD because of the patients' long-term history of smoking ${ }^{3)}$. Further, COPD itself may result in systemic inflammation. In particular, cerebrovascular and cardiovascular events are frequently observed ${ }^{4}$.

Univariate risk analyses of COPD have indicated that hyper-

Received: May 7, 2014, Accepted: May 13, 2014

${ }^{1}$ Address; Tokai University Tokyo Hospital

1-2-5 Yoyogi, Shibuya-ku Tokyo 151-0053, Japan

TEL: +81-3-3370-2321, FAX: +81-3-5354-5366

E-mail: akinoriebihara@hotmail.com tension, diabetes, obesity, and dyslipidemia are risk factors for ischemic heart disease. A multivariate analysis adjusted for all remaining factors showed that COPD remained an independent risk factor ${ }^{5)}$. COPD, stroke, chronic kidney disease, and diabetes are the most common comorbidities in patients with chronic heart failure ${ }^{6}$. COPD patients have a high prevalence of cardiovascular disease, higher than expected given their age and the coexistence of classic cardiovascular risk factors ${ }^{5}$.

It is known that the cause of death is determined by the respiratory disease in patients with severe and very severe disease, but in patients with mild and moderate disease deaths due to cancer and cardiovascular disease are more frequent ${ }^{7}$. Previous reports have analyzed the intima media thickness (IMT) of COPD patients using carotid artery ultrasound. It seems likely that tobacco smoke plays an important role in the development of cardiovascular disease in COPD and, notably, the carotid IMT in a group of healthy Japanese men was shown to be significantly increased in individuals who smoked and these individuals also had greater airflow limitation than matched healthy smokers and non-smokers ${ }^{8)}$. Smokers with a spirometric-based diagnosis of COPD may thus have evidence of subclinical atherosclerosis independent of tobacco smoking. 
Carotid artery IMT is associated with cardiovascular risk factors and prevalent cardiovascular disease, and is predictive of cardiovascular events. ${ }^{9-12)}$

We have previously reported that early exposure to tobacco smoke significantly increases the prevalence of $\mathrm{COPD}^{13)}$. As a follow-up, we hypothesized that COPD arising from early exposure to tobacco smoke may cause an increase in IMT that is correlated with the increase in the prevalence of smoke-related vascular comorbidities such as cardiovascular and cerebrovascular diseases.

\section{Materials and Methods}

This prospective, observational study of a consecutive cohort of COPD patients was carried out between 2009 and 2013. All patients provided their written informed consent at the Erimo clinic. Patients underwent spirometry (GRAPH junior HI-101: Chest Inc. Japan) and completed a self-report questionnaire on their clinical history and COPD risk factors including exposure to tobacco smoke and age of smoking initiation. COPD was diagnosed using the Global Initiative for Chronic Obstructive Lung Disease (GOLD) guidelines. Because we were unable to perform airway reversibility testing, we used the following four questions on the questionnaire in the same order as in the NICE study ${ }^{14)}$ : (1) During the past 12 months have you had more than one episode of waking at night or early in the morning as a result of spasmodic breathlessness even when you did not have a cold? (2) During the past 12 months have you had more than one episode of waking at night or early in the morning as a result of wheezing or whistling in the chest even when you did not have a cold? (3) Have you ever been told by a doctor that you had bronchial asthma? (4) Were you ever diagnosed as having asthma during your childhood? An affirmative response to any combination of these questions defined the patient as having possible asthma. The remaining patients with airflow limitation were considered to have COPD.

We defined severe smoking as more than 40 pack years and moderate exposure to tobacco smoke as 20-40 pack years. Pack years were calculated based on the number of cigarettes smoked per day and the number of years. For example, 1 pack year is equal to smoking 1 pack per day for 1 year or 2 packs per day for half a year.

Patients with airflow limitation were stratified by age, sex, and smoking status.

We divided potential subjects from the Erimo town clinic into three groups:

Group 1) Patients with a history of COPD and early exposure to tobacco smoke.

Group 2) Patients with a history of COPD and non-early exposure to tobacco smoke.

Group 3) Non-COPD patients with other diseases (diabetes, hypertension, or hyperlipidemia)

We defined early smoke exposure as habitual smoking starting before age 20. Cholesterol levels (LDL and HDL) and HbA1c were determined by blood test.

IMT was measured by ultrasonography using the longitudinal axis of the common carotid arteries. Segmentation of the intimamedia complex of the common carotid artery is important for the evaluation of the IMT on B-mode ultrasound images (Toshiba, Aplio(tm) 300 with 17" LCD color monitor).

\section{Statistical Analysis}

Statistical analyses were performed using SPSS v19.0 (IBM SPSS, Inc.). General analyses were performed using an unpaired $t$ test for continuous variables and a $\chi^{2}$ test for categorical variables. The standard $P<0.05$ significance level was used. All values in the text and tables are given as mean \pm standard deviation $(\mathrm{SD})$.

\section{Results}

A total of 152 subjects (mean age $72 \pm 10$ years) were enrolled in the study. Table 1 shows the patient characteristics. Group 1 included 41 subjects ( 32 men, 9 women; mean age $68 \pm 9$ years); Group 2 included 80 subjects (62 men, 18 women; mean age 71 \pm 11 years); and Group 3 included 31 subjects (18 men, 13 women; mean age $69 \pm 10$ years). In Group 1, 23 subjects did not experience any complications, 3 had diabetes, 8 hypertension, 3 hyperlipidemia, and 4 experienced other complications. In Group 2, 58 subjects did not have any complications, 5 had diabetes, 11 hypertension, 1 hyperlipidemia, and 5 other complications. In Group 3, 7 patients had diabetes, 12 hypertension, 3 hyperlipidemia, and 9 other complications. Hypertension was the most common complication followed by diabetes mellitus and hyperlipidemia. Figure 1 shows the frequency of all complications and Figure 2 shows the frequency of other diseases. The most common complication in both groups was hypertension. A total of $73 \%$ of patients in the COPD group that did not report early exposure to smoke did not experience complications compared with $56 \%$ of patients in the COPD group.

As shown in Table 2, HbA1c levels (JDS\%) in Groups 1, 2, and 3 were $6.6 \pm 0.8 \%, 7.3 \pm 0.9 \%$, and $6.9 \pm 0.9 \%$, respectively. Total cholesterol levels were $215 \pm 27 \mathrm{mg} / \mathrm{dL}, 199 \pm 20$ $\mathrm{mg} / \mathrm{dL}$, and $212 \pm 24 \mathrm{mg} / \mathrm{dL}$, respectively. Low-density lipopro-

Table 1 Patients Characteristics

In all groups, hypertension is the most common complication, followed by diabetes mellitus and hyperlipidemia.

\begin{tabular}{|c|ccc|}
\hline & G1 & G2 & G3 \\
& $\mathrm{n}=41$ & $\mathrm{n}=80$ & $\mathrm{n}=31$ \\
\hline gender (male) & 32 & 62 & 18 \\
age (year \pm SD) & $68 \pm 9$ & $71 \pm 11$ & $69 \pm 10$ \\
Complications & & & \\
non & 23 & 58 & - \\
DM & 3 & 5 & 7 \\
HTN & 8 & 11 & 12 \\
HL & 3 & 1 & 3 \\
others; & & & \\
DM + HTN & 1 & 1 & 2 \\
DM + HL & 1 & 0 & 0 \\
HTN + HL & 0 & 1 & 2 \\
DM + HTN + HL & 2 & 3 & 5 \\
\hline \multicolumn{2}{c}{} & \multicolumn{2}{c}{ (Number of patients) }
\end{tabular}




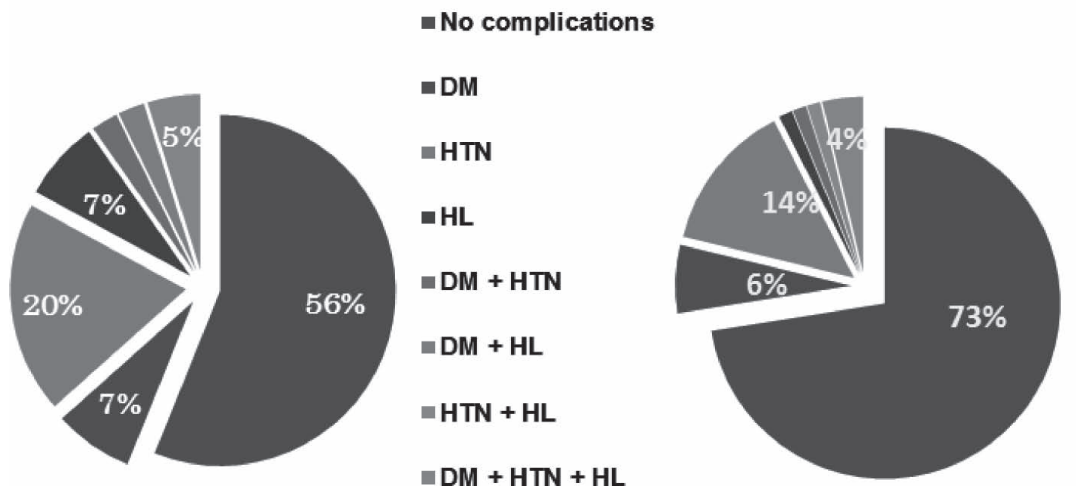

Fig. 1 The Rate of complications in Group 1 and Group 2.

The most common complication was hypertension in both groups. The rate of no complications was $73 \%$ in the COPD group without early exposure, but it was $56 \%$ in the COPD group with early exposure.

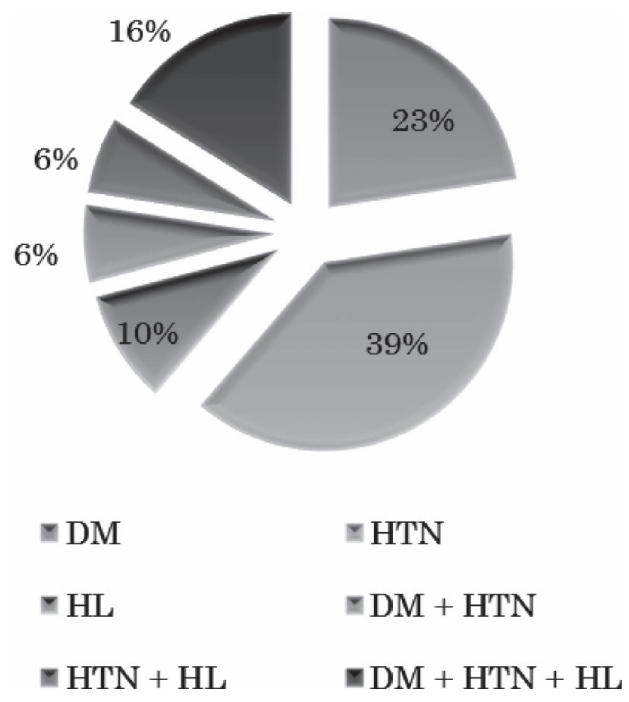

Fig. 2 The rate of other diseases in Group 3.

Hypertension is the most common (39\%), and diabetes mellitus is second $(29 \%)$. Combination of hypertension, hyperlipidemia and diabetes is third $(16 \%)$.

Table $2 \mathrm{HbA} 1 \mathrm{c}$ and cholesterol levels

There was no significant difference between three groups.

\begin{tabular}{|c|c|c|c|c|}
\hline & $\begin{array}{c}\text { HbA1c } \\
(\mathrm{JDS} \%)\end{array}$ & $\begin{array}{c}\mathrm{TC} \\
(\mathrm{mg} / \mathrm{dl})\end{array}$ & $\begin{array}{c}\mathrm{LDL} \\
(\mathrm{mg} / \mathrm{dl})\end{array}$ & $\begin{array}{c}\mathrm{HDL} \\
(\mathrm{mg} / \mathrm{dl})\end{array}$ \\
\hline $\mathrm{G} 1$ & $6.6 \pm 0.8$ & $215 \pm 27$ & $123 \pm 26$ & $43 \pm 16$ \\
\hline $\mathrm{G} 2$ & $7.3 \pm 0.9$ & $199 \pm 20$ & $106 \pm 31$ & $49 \pm 19$ \\
\hline $\mathrm{G} 3$ & $6.9 \pm 0.9$ & $212 \pm 24$ & $113 \pm 19$ & $42 \pm 22$ \\
\hline
\end{tabular}

tein levels were $123 \pm 26 \mathrm{mg} / \mathrm{dL}, 106 \pm 31 \mathrm{mg} / \mathrm{dL}$, and $113 \pm$ $19 \mathrm{mg} / \mathrm{dL}$, and high-density lipoprotein levels were $43 \pm 16 \mathrm{mg} /$ $\mathrm{dL}, 49 \pm 19 \mathrm{mg} / \mathrm{dL}$, and $42 \pm 22 \mathrm{mg} / \mathrm{dL}$, respectively. There were no significant differences among the three groups.

The maximum IMT values in Groups 1, 2, and 3 were $1.62 \pm$ $0.35 \mathrm{~mm}, 1.46 \pm 0.43 \mathrm{~mm}$, and $1.32 \pm 0.26 \mathrm{~mm}$, respectively.

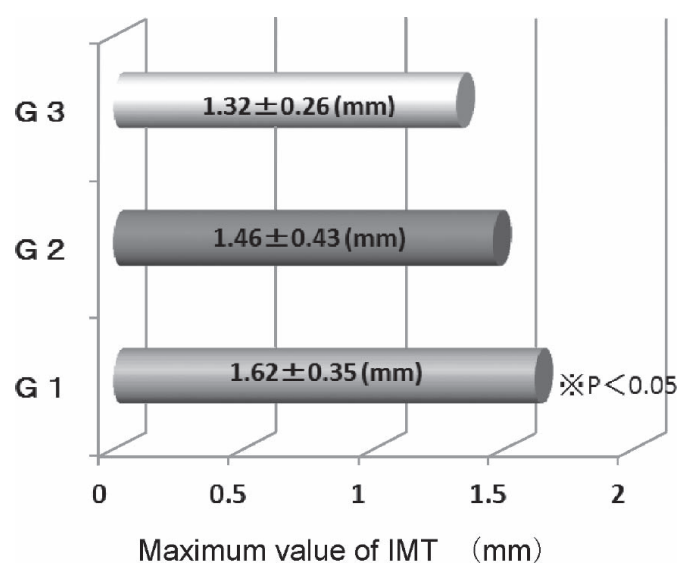

Fig. 3 Maximum value of IMT for each group. ※significantly different from Group 3.

The groups with a history of COPD tended to have higher maximum IMT values than the group without COPD. Furthermore, Group 1 had the highest IMT value (Fig. 3).

\section{Discussion}

COPD is a major cause of morbidity and mortality worldwide $^{15)}$. Atherosclerotic changes have been identified in patients with early exposure to smoke, which may increase the likelihood of smoke-related vascular comorbidities such as cardiovascular and cerebrovascular diseases ${ }^{5}$. There is increasing awareness that the inflammatory state associated with COPD is not confined to the lungs but also involves the systemic circulation and can affect non-pulmonary organs ${ }^{16)}$. In this study, we compared the incidence of diseases such as diabetes, hypertension, and hyperlipidemia with the max-IMT measurement, which is considered an arteriosclerotic index for COPD. In general, hypertension and diabetes are regarded as diseases that promote arteriosclerosis by inducing vascular endothelial cell death ${ }^{17}$.

In the present study, we attempted to ascertain the degree of 
arteriosclerosis in patients with COPD concurrent with hyperlipidemia, hypertension, and diabetes treatment. It is well known that diabetes is a common cause of arteriosclerosis, but in this study, we were interested in identifying severe arteriosclerotic changes that occurred following long-term exposure to tobacco smoke among patients with hyperlipidemia, hypertension, and diabetes. The groups with a history of COPD tended to have higher maximum IMT values that the group without COPD. Furthermore, Group 1 had the highest IMT value. Early smoke exposure may thus play an important role in the development of arteriosclerosis.

COPD is now the third leading cause of death in the United States and it is the only chronic disease for which attributable mortality continues to rise ${ }^{18)}$. Risk factors for COPD include tobacco smoking, advanced age, and environmental pollution ${ }^{19)}$. Of these, tobacco smoking is the most important and it is also a major risk factor for coronary artery disease. Lifetime smokers have a $15-50 \%$ chance of developing $\mathrm{COPD}^{20,21)}$, and recent studies place the population-attributable risk of smokers developing the disease at $51-70 \%{ }^{22}$. The INTERHEART study, a case-control study matched for age and sex, showed that smoking accounted for $36 \%$ of the population-attributable risk of a first myocardial infarction ${ }^{23}$. Mechanisms underlying the association between atherosclerosis and COPD are not yet well understood. However, chronic systemic inflammation and IMT thickening are possible contributors ${ }^{24)}$. The results of the present study agree with these previous observations.

Further studies are needed to elucidate the precise magnitude of the increase in risk associated with these comorbidities of COPD and early smoke exposure.

\section{Acknowledgements}

The authors wish to thank staff of Erimo town clinic and Health Division of Erimo Town. This study was funded by grant from the Japan Society of Health Evaluation and Promotion, and Grants-in-Aid for Scientific Research (No. 24659246).

\section{The authors state that they have no Conflict of Interest (COI).}

\section{REFERENCES}

1) Murray CJ, Lopez AD: Alternative projections of mortality and disability by cause 1990-2020: Global Burden of Disease Study. Lancet: 349: 1498-1504, 1997.

2) Gourab C, Roberto R, William M: Comorbidities and systemic effects of chronic obstructive pulmonary disease. Clin Chest Med: 35: 101-130, 1997.

3) Nalaka S, Gooneratne N: Chronic obstructive pulmonary disease diagnosis and management in older adults. $\mathrm{J}$ of the American Geriatrics Society: 58: 153-162, 2010.

4) Johanna R, Laura C, Christopher J, et al.: Chronic obstructive pulmonary disease Prevalence of major comorbidities in subjects with COPD and incidence of myocardial infarction and stroke: a comprehensive analysis using data from primary care. Thorax: 65: 956-962, 2010.

5) Pilar L, Jose L, Jose M, et al.: Chronic obstructive pulmonary disease as a cardiovascular risk factor. Results of a case-control study (CONSISTE study). Int J Chron Obstruct Pulmon: 7: 679686, 2010.

6) Vincent M. Deursen V, Renato U, et al.: Co-morbidities in patients with heart failure: an analysis of the European Heart Failure Pilot Survey Eur J Heart Fail: 16: 03-11, 2014.

7) Frampton J: QVA149 (Indacaterol/Glycopyrronium Fixed-dose combination): A review of its use in patients with chronic obstructive pulmonary disease. Drugs: 74: 465-488, 2014.

8) Iwamoto H, Yokoyama A, Kitahara Y, et al.: Airflow limitation in smokers is associated with subclinical atherosclerosis. Am J Respir Crit Care Med: 79: 35-40, 2009.

9) Bots M, Breslau P, Briet E, et al.: Cardiovascular determinants of carotid artery disease: the Rotterdam Elderly Study. Stroke: 19: 717-720, 1992.

10) Chambless L, Folsom A, Clegg L, et al.: Carotid wall thickness is predictive of incident clinical stroke: the Atherosclerosis Risk in Communities (ARIC) study. Am J Epidemiol: 151: 478-487, 2000.

11) Hodis H, Mack W, LaBree L, et al.: The role of carotid arterial intima-media thickness in predicting clinical coronary events. Ann Intern Med: 128: 262-269, 1998.

12) O'Leary D, Polak J, Kronmal R, et al.: Carotid-artery intima and media thickness as a risk factor for myocardial infarction and stroke in older adults: Cardiovascular Health Study Collaborative Research Group. N Engl J Med: 340: 14-22, 1999.

13) Ebihara A, Nagai A, Nakano $T$, et al.: The Influence of early exposure to tobacco smoke for pulmonary disease. Health Evaluation and Promotion: 39; 540-544, 2012.

14) Fukuchi $Y$, Nishimura $M$, Ichinose $M$, et al.: COPD in Japan: the Nippon COPD Epidemiology study. Respirology: 9: 458-465, 2012.

15) Nicola A. Management and burden of COPD in the Middle East and North Africa: the BREATHE study. Epidemiology: 1-2, 2012.

16) Surya P, Mark T: Chronic obstructive pulmonary disease and cardiovascular disease. Transl Res: 162: 237-251, 2012.

17) Bartolo J, Chan M, Bennett S, et al.: TNF-related apoptosisinducing ligand (TRAIL) protects against diabetes and atherosclerosis in Apoe mice. Diabetologia: 54: 3157-3167, 2012.

18) Minino A, MurphyS, Xu J, et al.: Deaths: final data for 2008. Natl Vital Stat Rep: 59: 120-26, 2012.

19) Decramer M, Janssens W, Miravitlles M: Chronic obstructive pulmonary disease. Lancet: 379: 1341-1351, 2012.

20) Lundback B, Lindberg A, Lindstrom M, et al.: Not 15 but $50 \%$ of smokers develop COPD? Report from the obstructive lung disease in northern sweden studies. Respir Med: 97: 115-122, 2003.

21) Ezzati M, Lopez A: Estimates of global mortality attributable to smoking in 2000. Lancet: 362: 847-852, 2003.

22) Wilson $D$, Adams R, Appleton S, et al.: Difficulties identifying and targeting COPD and population-attributable risk of smoking for COPD: a population study. Chest: 128: 2035-2042, 2003.

23) Salim Y, Steven H, Stephanie O, et al.: Effect of potentially modifiable risk factors associated with myocardial infarction in 52 countries (the INTERHEART study): case-control study. Lancet: 364: 937-52, 2004.

24) Fimognari F, Scarlata S, Conte M, et al.: Mechanisms of atherothrombosis in chronic obstructive pulmonary disease. Int J Chron Obstruct Pulmon Dis: 8: 89-96, 2008. 